\title{
Micro-dissection and integration of long and short reads to create a robust catalog of kidney compartment-specific isoforms
}

Ridvan Eksi ${ }^{1}$, Daiyao $\mathrm{Yi}^{1}$. Hongyang Li ${ }^{1}$, Bradley Godfrey ${ }^{2}$, Lisa R. Mathew ${ }^{3}$, Christopher L. O’Connor ${ }^{2}$, Markus Bitzer ${ }^{2}$, Matthias Kretzler ${ }^{1,2}$, Rajasree Menon ${ }^{1,2^{*}}$, Yuanfang Guan ${ }^{1,2^{*}}$

${ }^{1}$ Department of Computational Medicine and Bioinformatics, University of Michigan, 100 Washtenaw Ave, Ann Arbor, MI 48109-2218, USA

${ }^{2}$ Department of Internal Medicine, University of Michigan, 1500 E Medical Center Dr, Ann Arbor, MI 48109, USA

${ }^{3}$ Harvard College, MA, 02138, USA

* Corresponding: gyuanfan@umich.edu, rajmenon@umich.edu

Keywords: Alternative spliced isoforms, kidney transcriptome, PacBio Iso-seq sequencing, long reads, novel transcripts

\begin{abstract}
Studying isoform expression at the microscopic level has always been a challenging task. A classical example is kidney, where glomerular and tubulo-insterstitial compartments carry out drastically different physiological functions and thus presumably their isoform expression also differs. We aim at developing an experimental and computational pipeline for identifying isoforms at microscopic structure-level. We microdissed glomerular and tubulo-interstitial compartments from healthy human kidney tissues from two cohorts. The two compartments were separately sequenced with the PacBio RS II platform. These transcripts were then validated using transcripts of the same samples by the traditional Illumina RNA-Seq protocol, distinct Illumina RNA-Seq short reads from European Renal cDNA Bank (ERCB) samples, and annotated GENCODE transcript list, thus identifying novel transcripts. We identified 14,739 and 14,259 annotated transcripts, and 17,268 and 13,118 potentially novel transcripts in the glomerular and tubulo-interstitial compartments, respectively. Of note, relying solely on either short or long reads would have resulted in many erroneous identifications. We identified distinct pathways involved in glomerular and tubulointerstitial compartments at the isoform level.We demonstrated the possibility of micro-dissecting a tissue, incorporating both long- and short-read sequencing to identify isoforms for each compartment.
\end{abstract}

\section{Keywords}

transcript isoform, long-read RNA-seq, micro-dissect, kidney

\section{Introduction}

Gene expression patterns at the microscopic level can facilitate the understanding of the function of a tissue (Alberts et al. 2002). While single cell sequencing can now provide gene expression levels for individual cells (Eberwine et al. 2014), the assignment to respective microscopic structure remains a challenging task. Furthermore, the majority of the experimental protocols use short reads in single cell 
sequencing. While short reads can provide some information about the isoform expression levels, the assignment is not conclusive.

We aim at addressing the above challenge by developing an integrative protocol to micro-dissect microscopic structures of a tissue, followed by both short-read sequencing and long-read sequencing to identify isoforms, and particularly novel isoforms, in these microscopic structures. We used kidneys as a model tissue, due to both disease relevance and clear delineation of two types of compartments in the kidney: glomerular and tubulo-interstitial compartments. The glomeruli function to filter the blood and extra fluid and wastes pass into the tubule and form urine. Chronic kidney disease is prevalent in 14.5\% of the US population (Saran et al. 2020). Kidney diseases are traditionally classified based on etiology and pathological appearances, but in reality, kidney diseases are a collection of diseases with unique mechanisms, progression rates, and therapeutic responses despite sharing similar histopathological appearances (Lea-Henry et al. 2018). Micro-dissected renal biopsy specimens are rich sources for capturing the gene expression data of distinct compartments of the kidney glomerular and tubulo-interstitial compartments. Kidney transcriptomic studies typically use generic transcriptome databases such as Ensembl (Howe et al. 2021), GENCODE (Frankish et al. 2021), or NCBI (Frankish et al. 2021; O'Leary et al. 2016), which are incomplete and miss some of the critical kidney-specific transcripts. Moreover, when we use the complete set of annotated transcripts, some short reads from expressed annotated transcripts may be assigned to very similar non-expressed annotated transcripts. This resulted in miscalculated Fragments Per Kilobase of transcript per Million mapped reads values (FPKM) and power-loss in differential expression analysis, which can be remedied by the long reads. In addition, alternative splicing (AS) exponentially increases the information content of genomes by producing multiple transcripts from a single gene, further complicating the picture. Approximately $95 \%$ of multi-exonic human genes undergo AS, producing more than 100,000 distinct transcripts from approximately 20,000 protein-coding genes (McManus and Graveley 2011). Therefore, a complete and reliable database of transcript isoforms that is specifically expressed in the kidney using accurate long reads will improve the characterization of new mechanisms, biomarkers, and therapeutic targets.

Recent progress in single-molecule long-read sequencing has provided powerful new tools for researchers to resolve the previous inaccuracies of short-read RNA sequencing (Wang et al. 2009; Steijger et al. 2013; Hosoya et al. 2018). Pacific Biosciences (PacBio) has developed a technique based on Single Molecule Real Time sequencing (SMRT). The sequencing length of the PacBio RS II platform used in this study is $10 \mathrm{~kb}$, covering the entire size of most eukaryotic transcripts. This capability allows the sequencing of long sections of genomic DNAs or transcripts without fragmentation or PCR amplification. Thus, PacBio's full-length or nearly full-length transcripts eliminate the need for transcript assembly for downstream analysis. However, one limitation of the PacBio platform is its relatively high sequence error rate, but fortunately, these errors are randomly distributed across the reads. Recently improved read-length and base-calling algorithms of the PacBio SMRT analysis platform and the use of circular molecules have mitigated this error rate. When the read length exceeds the length of the cDNA template, each base pair is covered on both strands multiple times, and these low-quality base calls are aggregated to derive high-quality, single-molecule Reads of Inserts (ROIs). The PacBio long-read transcriptome sequencing platform (Iso-Seq) has been successfully applied to human and other species, and it has shown that the use of Iso-seq has a significant advantage over short-read RNA-Seq methods for identifying novel isoforms, detecting AS and gene fusion events (Sharon et al. 2013; Weirather et al. 2015; O'Grady et al. 2016; Gonzalez-Ibeas et al. 2016).

In this study, we examined human kidney cortex tissue to study the overall transcriptome of glomerular (hereafter referred to as "glo") and tubulo-interstitial (hereafter referred to as "tub") compartments using both long reads from PacBio Iso-Seq platform and RNA-seq short reads from 
Illumina platform. To validate full-length transcripts sequenced by PacBio, we used short reads to generate high-quality sets of transcript isoforms from two distinct clinical sources. Then, we compared the confirmed transcript isoforms to the set of known transcript isoforms in GENCODE and provided the final list of expressed transcript isoforms and their annotation status for researchers in downstream kidney transcriptome studies. Using these analyses, we identified a large number of novel transcript isoforms and pathways in these two kidney compartments, creating an important resource for the kidney research community. Most importantly, the experimental and computational pipeline developed in this study is promising to be applied to other tissues to acquire the isoform catalog at microscopic levels.

\section{Materials and Methods}

\section{Ethics statement}

The study is approved by the University of Michigan Institutional Review Board (HUM00002468: Expression Analysis in Human Renal Disease). All participants have provided written consent to the study.

\section{RNA extraction from human kidney cortical tissue}

We used healthy human kidney cortex cores obtained from five patients who underwent tumor nephrectomies and 20 healthy samples from the European Renal cDNA Bank (ERCB) study (Schmid et al. 2006). The kidney cores were immediately placed in RNA. Later solution at $4^{\circ} \mathrm{C}$ for 12 to 24 hours and then stored at $-20^{\circ} \mathrm{C}$ (Schmid et al. 2006). Micro-dissection of glomerular and tubulo-interstitial compartments was performed as previously described (Ju et al. 2009; Kato et al. 2016).

\section{RNA library preparation/Sequencing using Illumina platform}

For Illumina RNA-seq runs, TapeStation (Agilent, Santa Clara, CA) assessed the RNA quality while following the manufacturer's recommended protocols. Samples with RNA Integrity Numbers (RINs) of 8 or higher were prepared using the Illumina TruSeq mRNA Sample Prep v2 kit (Catalog \#s RS-122-2001, RS-122-2002, Illumina, San Diego, CA) using manufacturer's recommended protocols. 0.1-3 $\mu$ g of total RNA was enriched for mRNA using a polyA purification, and the mRNA was then fragmented and copied into first strand cDNA using reverse transcriptase and random primers. The 3 prime ends of the cDNA were then adenylated, and the adapters were ligated. One such adapter was a six-nucleotide barcode unique to each sample allowing us to sequence more than one sample in each lane of a HiSeq flow cell (Illumina). The products were purified and enriched by polymerase chain reactions to create the final cDNA library. Final libraries were checked for quality and quantity by TapeStation (Agilent) and qPCR using Kapa's library quantification kit for Illumina Sequencing platforms (catalog \# KK4835, Kapa Biosystems, Wilmington MA) using manufacturer's recommended protocols. The libraries were clustered on the cBot (Illumina) and sequenced 4 samples per lane on a 50 cycle paired-end for tumor nephrectomy samples, and 1 sample per lane on a 100 cycle paired-end run for ERCB samples on a HiSeq 2000 (Illumina) in High Output mode using version 3 reagents according to manufacturer's recommended protocols.

\section{RNA sequencing with the PacBio platform}


For the PacBio library, equal proportions of RNA from the tumor nephrectomy samples were pooled to form $500 \mathrm{ng}$ of RNA for each compartment and processed for next-generation sequencing (NGS) library preparation. PacBio sequencing library preparation was done according to the manufacturer's recommendation for Isoform Sequencing using the Clontech SMARTer PCR cDNA synthesis kit and BluePippin Size-Selection System. cDNA SMRTbell templates were fractionated into $1 \mathrm{~kb}-2 \mathrm{~kb}, 2 \mathrm{~kb}-$ $3 \mathrm{~kb}, 3 \mathrm{~kb}-6 \mathrm{~kb}$, and $5 \mathrm{~kb}-10 \mathrm{~kb}$. Sixteen SMRT cells were used in total: two for each size fraction of both glomerular and tubulointerstitial compartments. Sequencing was performed on a Pacific Biosciences PacBio RSII by University of Michigan DNA Sequencing Core. Each glomerular and tubulo-interstitial compartment had eight sequencing cells, generating 132,240 and 125,047 SMRT CFLs respectively. These CFLs were supported by 206,415 and 232,845 SMRT Reads of Inserts (ROIs). These were determined to be full-length based on the apparent 5' and 3' cDNA primer sequences and polyA tail at 3' end.

\section{Sequence generation and alignments}

Illumina RNA-Seq reads were aligned and mapped using STAR (Dobin et al. 2013) version 2.5 to the human genome (hg19 assembly). STAR was run in a 2-pass mode with suggested parameters under "ENCODE options" heading in the STAR manual. Pacific Bioscience SMRT raw reads were initially processed using the Pacific Biosciences SMRT analysis software version 2.3.0. The polymerase reads were partitioned into sub-reads. Read of Inserts (ROI) were generated using the default number of polymerase full passes. The Iso-Seq classify tool was then used to separate the ROIs into full-length non-chimeric and non-full length reads. We defined full-length reads as containing 5' and 3' cDNA primers and polyA tails. Then, the Iso-Seq cluster tool was used to cluster all the full-length reads derived from the same transcript to produce the consensus full-length transcripts (CFLs). CFLs that were unpolished by Quiver were used in the rest of the analysis because it had been reported that Quiver polishing sometimes obscured the introns (O'Grady et al. 2016). SMRT CFLs were aligned and mapped to the human genome (hg19 assembly) using GMAP (Wu and Watanabe 2005). We kept reads mapping to a single location (argument $-\mathrm{n} 1$ ).

As the next phase of the analysis, the pipeline for identification of transcription start sites, splice junctions and transcription end sites were adapted from the TRIMD pipeline (Wu and Watanabe 2005; O'Grady et al. 2016). Single-exon CFLs were excluded from the following validation steps, as most of them were potential intronic fragments resulting from pre-processed mRNAs. Single-exon CFLs were added back to analysis before the collapse step.

\section{Identification of transcription start sites (TSS)}

CFL 5' end clusters were generated with CFL 5' ends mapping within 8 bp of each other. Only CFL isoforms whose 5' ends did not contain mismatches were used. A single TSS is determined for each of the clusters by calculating weighted (based on the number of SMRT reads for each start coordinate) averages of the start coordinates of CFLs within the cluster. These consensus TSS are considered validated if there is an annotated transcription start site within $10 \mathrm{bp}$ vicinity (Wu and Watanabe 2005). Annotated TSS are extracted from GENCODE comprehensive annotation set (version 24).

\section{Identification of splice junctions}


Splice junctions from Iso-Seq CFLs were identified using GMAP, and splice junctions for Illumina reads were identified with STAR (Dobin et al. 2013). Splice junctions from Iso-Seq CFLs are required to have at least 1 full-length read spanning it to be identified. A splice junction from an Iso-Seq CFL is marked as validated if at least 3 short reads are spanning it or if the junction is already annotated. Annotated junctions are extracted from GENCODE comprehensive annotation set (version 24).

\section{Identification of transcription end sites (TES)}

Illumina reads that have poly(A) tails were extracted from SAM alignment files. These putative reads with poly(A) tails are the reads that have a FLAG code as being first-of-pair, and either end with a run of at least five As, at least two of which are mismatched on plus strand or that start with a run of at least five Ts, at least two of which are mismatched on minus strand. The alignment position base next to the mismatched location was considered a candidate TES. TES that is within $8 \mathrm{bp}$ of each other were considered single candidate TES. The consensus TES coordinate was determined using a weighted average of putative $3^{\prime}$ ends based on a few short reads supporting each TES coordinate. TES are marked as validated either if there is an Illumina TES on the same strand within four bases upstream or ten bases downstream or if there is an annotated TES in the 10bp vicinity (O'Grady et al. 2016).

Iso-Seq CFL $3^{\prime}$ ends that align within 8 bp of each other on the genome are considered a single candidate TES. The CFL consensus TES were determined by calculating weighted averages of the end coordinates. Weights are determined by the number of PacBio consensus sequence reads ending at each coordinate. Only putative PacBio 3' end sites that are supported by at least three SMRT reads are kept.

\section{CFL validation and comparison to known annotations}

TSS, splice junction and transcription end sites of each glomerular CFLs are compared to coordinates extracted from short-reads from ERCB glomerular samples and annotated transcripts. For tubulointerstitial compartment, transcript features are compared to short-read RNA-seq data from matching tumor nephrectomy samples, and ERCB tubulo-interstitial samples separately. Iso-Seq CFL validation was done by validating every splice junction present in the CFL. An Iso-Seq CFL was considered validated if every splice junction was validated based on the criteria explained above. Two different short-read RNA-seq datasets for the tubulo-interstitial compartment were combined for this step. Single-exon CFLs were added to the set of validated multi-exon CFLs, pending further investigation based on their relative location to a known transcript. The set of validated multi-exon CFLs and single-exon CFLs were collapsed with the collapse_isoforms_by_sam.py script in the tofu package provided by PacBio, which is the developmental version of the official Iso-Seq protocol. Then, collapsed isoforms were compared to the GENCODE comprehensive set of annotations (version 24) with a cuffcompare tool from Tuxedo suite of tools (Roberts et al. 2011a, 2011b; Trapnell et al. 2013, 2010).

\section{Data Records}

The set of validated and collapsed isoform sequences with their corresponding annotation class based on cuffcompare tool can be found in "Glom_validated_transcripts.fa" (Data Citation 1) for glomerular compartment and in "Tubulo_validated_transcripts.fa" (Data Citation 2) for tubulo-interstitial compartment. Both files are in fasta format. The ID line for each sequence entry contains an internal transcript ID (PB.X.X), chromosome name, location, cuffcompare class code and associated gene's ENSEMBL gene id. 


\section{Technical Validation}

For short-read RNA-seq runs, only samples with 8 or higher RINs were used. For long-read RNA-seq with the PacBio RS II platform, equal proportions of RNA from the tumor nephrectomy samples were pooled to form $500 \mathrm{ng}$ of RNA for each compartment and processed for next-generation sequencing (NGS) library preparation. Throughout this study, every sample preparation step was done according to the manufacturer's recommendation.

\section{Results}

\section{Long-read sequencing analysis of kidney transcriptome}

In this study, we micro-dissected glomerular and tubulo-interstitial compartments from 25 healthy human kidney cortex core samples following (Ju et al. 2009; Kato et al. 2016) (detailed protocol please see methods). Among the 25 samples, five were from patients who underwent tumor nephrectomies and 20 healthy samples from the European Renal cDNA Bank (ERCB) study (Schmid et al. 2006). PacBio's Iso-seq protocol was used for library preparation and long-read sequencing, and PacBio's SMRT Portal was used for the initial data analyses. Each glomerular and tubulointerstitial compartment had eight sequencing cells, from which we generated 132,240 (glo) and 125,047 (tub) SMRT consensus full-length transcripts (CFLs), validated by two high-quality sets of transcript isoforms from two distinct clinical sources based on short reads (Figure 1). These were determined to be full-length based on the apparent 5' and 3' cDNA primer sequences and polyA tail at 3' end. Glomerular CFLs ranged from 300 to 27,890 bases in length with a mean of 2,446 bases. Tubulointerstitial CFLs ranged from 300 to 22,765 bases in length with a mean of 2,862 bases. Size fractionation of the library before sequencing reduced the bias toward shorter transcripts. These CFLs were mapped to the human genome (hg19 assembly) using GMAP (Wu and Watanabe 2005). Approximately $98 \%$ of the CFLs from both compartments were uniquely mapped to the genome.

We further identified 42,896 and 38,831 putative transcription start sites (TSS) from PacBio glomerular and tubulointerstitial multi-exon CFLs respectively (see "Methods"). As demonstrated in Figure 2 A-B using sample gene AIF5, we could not reliably infer TSS from Illumina short reads; our validation criteria relied only on annotated TSS: Top tracks in the figure $2 \mathrm{a}, 2 \mathrm{~b}$ and $2 \mathrm{c}$ are illumina short reads. Just by looking at the read coverage of illumina reads, we cannot pinpoint a location for the TSS, meaning there is not a cliff where read coverage suddenly starts. As a result, we only used TSS that exists in the annotation file. Gene AIF5 has multiple CFLs with varying start positions. During transcript collapsing the CFLs that are identical other than their start positions are merged, and the start coordinate of CFL with the longest 5' end is taken as the true 5' end for the merged transcript. For gene AIF5, we identified 13 TSS and 6 of them were more than 10bp away from the annotated TSS.

Through mapping PacBio multi-exon CFLs to the genome, we identified 171,742 and 185,185 splice junctions in glomerular CFLs and tubulo-interstitial CFLs, respectively. Each splice junction has at least one full-length SMRT read spanning them. We further utilized short reads to validate the new splice junction findings. A validated set of splice junctions is the union of annotated junctions and splice junctions that have at least three short reads spanning them from Illumina RNA-Seq data. For glomerular compartments, approximately $65 \%$ of PacBio junctions were validated. In tubulo-interstitial compartments, using the shallower tumor nephrectomy Illumina RNA-seq data, we validated approximately $64 \%$ of all PacBio tubular splice junctions. For example, for tubulo-interstitial, the deeper 
ERCB RNA-seq data allowed us to validate an additional 8,824 - 3,105 = 5,719 novel splice junctions (Table 1).

We further identified 24,625 and 26,260 putative transcription end sites (TES) in PacBio glomerular and tubulo-interstitial multi-exon CFLs through the process explained in Methods. Then, we extracted short reads containing polyA reads from Illumina RNA-Seq data and extracted polyA site coordinates from those reads. We considered putative TES from PacBio validated if they were near either an annotated TES or a polyA site extracted from short reads. Although it is not as prevalent as the 5' ends, we have a high variation in the transcript end sites extracted from PacBio CFLs (Figure 2C), and this variation is incompletely captured by short-reads as we have a limited number of reads with polyA tails. We decided to provide CFLs present in the sample with their original 3' end locations to give readers a choice to process CFLs with different 3' UTR lengths based on their study objective.

\section{CFL validation and collapsing into final structures}

Since exact TSS and TES coordinates do not agree well with annotated coordinates, we did not use these transcript features in whole CFL validation. Our validation criterion required the CFL to have all its junctions validated either by short read support or by annotation. With this criterion, 53,540 tubulointerstitial multi-exon CFLs and 47,785 glomerular multi-exon CFLs were marked as validated. Any two isoforms that differed on the $3^{\prime}$ end by more than $100 \mathrm{bp}$ (a defined threshold by PacBio) were considered different isoforms. If two isoforms differed only by their $5^{\prime}$ ends, meaning one isoform had 0 , 1 , or more $5^{\prime}$ exons than the others but all remaining exons agreed, then the shorter isoform was considered identical to the longer one, and it was collapsed into the longer isoforms. After collapsing, 45,778 and 58,378 isoforms were formed from tubulointerstitial and glomerular tissue.

\section{Comparison of validated isoforms to annotated transcripts}

We compared the list of collapsed CFLs to the annotated set of transcripts from GENCODE version 24. The comparison was made with the cuffcompare tool in Cufflinks which classifies each input transcript into twelve distinct classes based on their overlap with an annotated transcript. The seven most prevalent classes and the numbers of collapsed transcripts from tubulo-interstitial and glomerular compartments belonging to each of these seven classes are shown in Table 2. "Complete match of intron chain with an annotated transcript," and "Contained within a reference transcript" classes comprise the set of expressed annotated transcripts in the sample. The set of transcripts that belonged to "A transflag falling entirely within a reference intron" class was discarded. These are single exon transcripts, which are most likely by-products of the intron decay process (Sharon et al. 2013). Other single exon transcript classes are "Single exon transfrag overlapping a reference exon and at least $10 \mathrm{bp}$ of a reference intron" and "Exonic overlap with reference on the opposite strand class", which were also discarded. These are possible pre-mRNA fragments that were pulled down due to the inefficient polyA selection step. The class of "Potentially novel isoforms" includes transcripts that share at least one junction with an annotated transcript and have junctions that do not occur in any annotated transcript. If junctions occur in the annotated transcript, their combination is novel. Again, readers should be cautious about the exact location of TSS in these transcripts as transcripts may be truncated.

The class of "Intergenic transcripts" includes transcripts that map to an intergenic location. Most of these intergenic transcripts are single exon transcripts, more likely regulatory non-coding mRNAs. The two sets of validated and collapsed isoform sequences with their corresponding annotation class are provided in the Supplementary Files. 


\section{Example novel isoforms and novel intergenic transcripts identified by Pac-bio long-read sequencing}

NPHS2 (podocin) is a protein-coding gene located on chromosome 1. This gene has 8 exons and has two protein coding splice variants according to the GENCODE database. NCBI's RefSeq lists three other predicted protein-coding splice variants. Our validated set of glomerular isoforms has 11 different splice variants for this gene (Figure 3). Two of these precisely match variants in GENCODE, and the other two match to the two predicted splice variants in RefSeq. Four other splice variants have the same first and last exons. However, their different combinations make them novel variants. The remaining three novel splice variants have alternative end sites. Among the seven novel splice variants, there is one novel junction, which is supported by multiple short reads.

Among the final set of isoforms, there are 4,208 and 9,501 intergenic transcripts from tubulo-interstitial and glomerular compartments. The majority of these transcripts have single exons, and therefore their junctions do not require validation. There are 76 and 55 multi-exon intergenic transcripts in tubulointerstitial and glomerular compartments. Because they passed the validation criteria, all the junctions in these transcripts are supported by multiple short reads.

\section{Comparison of the expressed set of annotated transcripts in glomerular and tubulo-interstitial compartments}

Glomerular and tubulo-interstitial compartments are expected to have distinct transcriptome profiles. In this section, we compared the set of expressed annotated transcripts from each compartment. 3,993 and 13,536 annotated transcripts are expressed in these compartments, of which 8,198 are common transcripts (Figure 4A). We performed KEGG pathway enrichment on the transcripts that are uniquely expressed in each compartment. A full list of enriched pathways is in Supplementary Table 1. Figure 4B shows the top 3 enriched pathways for each compartment. Glomerular-only expressed transcripts are enriched for Non-alcoholic fatty liver disease (NAFLD), RAP1 signaling pathway, and ubiquitin-proteosome pathway. Tubulointerstitial transcripts are enriched in metabolic, ribosomal, and aldosterone-induced sodium reabsorption pathways.

\section{Discussion}

In this study, we aim at exploring the potential of using combined information from long- and short-read sequencing information to create catalogs of expressed isoforms for microscopic structures. Towards this goal, we used human kidney tissues, which consists of structures of distinct physiological functions. By micro-dissecting glomerular and tubular compartments, we generated biosamples whose half goes to short-read sequencing and the other half goes to long-read sequencing. We designed a pipeline to first use long reads to identify all putative isoforms, and use the short-reads to confirm their relevant junctions. This approach is distinct from current single-cell sequencing efforts or traditional bulk sequencing, in that a microscopic structure carrying out specific physiological function is the study target.

We found tens of thousands of novel transcripts in glomerular and tubular compartments validated by both long and short read sequencing, creating a rich repertoire of transcripts for future functional studies. Preliminary analysis of these transcripts demonstrates drastically different pathway enrichment between the glomeruli and the tubular compartment, supporting the success of this approach. 
We envision several future directions of investigation. First, such experimental protocols of microscopic investigation of isoforms can be carried out in other tissues. Second, many novel transcripts are identified in the study, connecting them to isoform function prediction methods, e.g., (Li et al. 2014) will help us to elucidate the activated pathways of these isoforms. Third, connecting the expression patterns from these microscopic structures to single-cell level sequencing can help us understand the variability of the expression patterns of these isoforms. Fourth, the focus of this paper is on health tissues. It will be interesting to study the isoform changes between the healthy state and the disease state of the same microscopic structures. We foresee all these will be exciting opportunities when such microscopic dissection followed by isoform identification with long and short-reads will become well accepted in the medical field.

\section{Acknowledgements}

This work is supported by NSF 1452656, NIH R35GM133346.

\section{Competing interests}

YG receives personal payment from Eli Lilly and Company, which may have sponsored certain aspects of the study to MK.

\section{Author Contributions}

Manuscript: YG and RE. Computational experiments and data analytics: RE. DY made figures. Wet lab experiments: BG. YG and RM supervised this study. All authors contributed to the writing of the manuscript and approved the final manuscript.

\section{Data availability}

1. Eksi, Ridvan Figshare https://figshare.com/articles/Glom validated transcripts fa/7305023

2. Eksi, Ridvan Figshare https://figshare.com/articles/Tubulo validated transcripts fa/7305029

3. https://osf.io/nhkbr/?view_only=6b561d2875f0449fa8e64dc841539780 

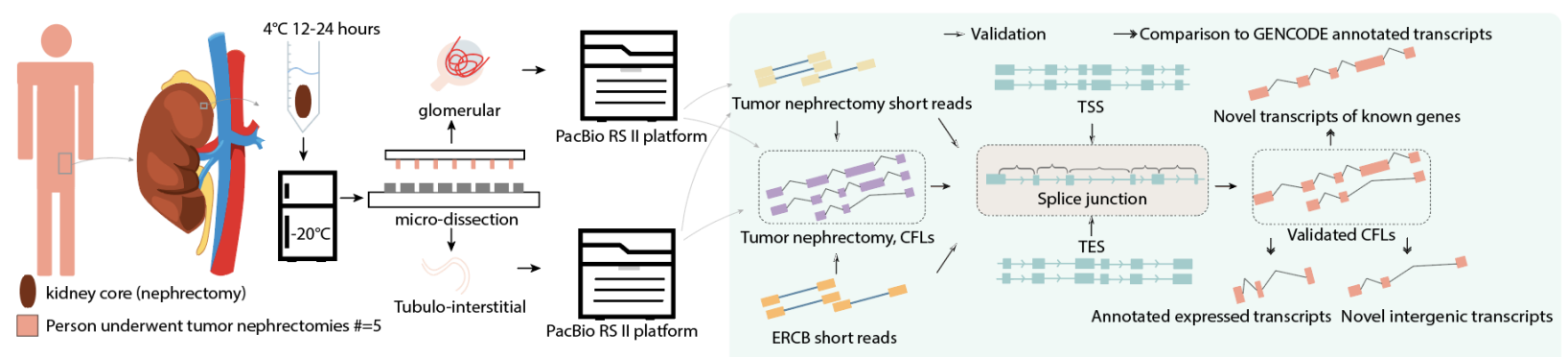

Figure 1: Overall study design. The features of every multi-exon Consensus Full-Length transcripts (CFL's) found in PacBio reads were validated through Illumina short reads from two different RNA-seq datasets. Whole CFLs were then validated at every splice junction before comparison to GENCODE annotation. 


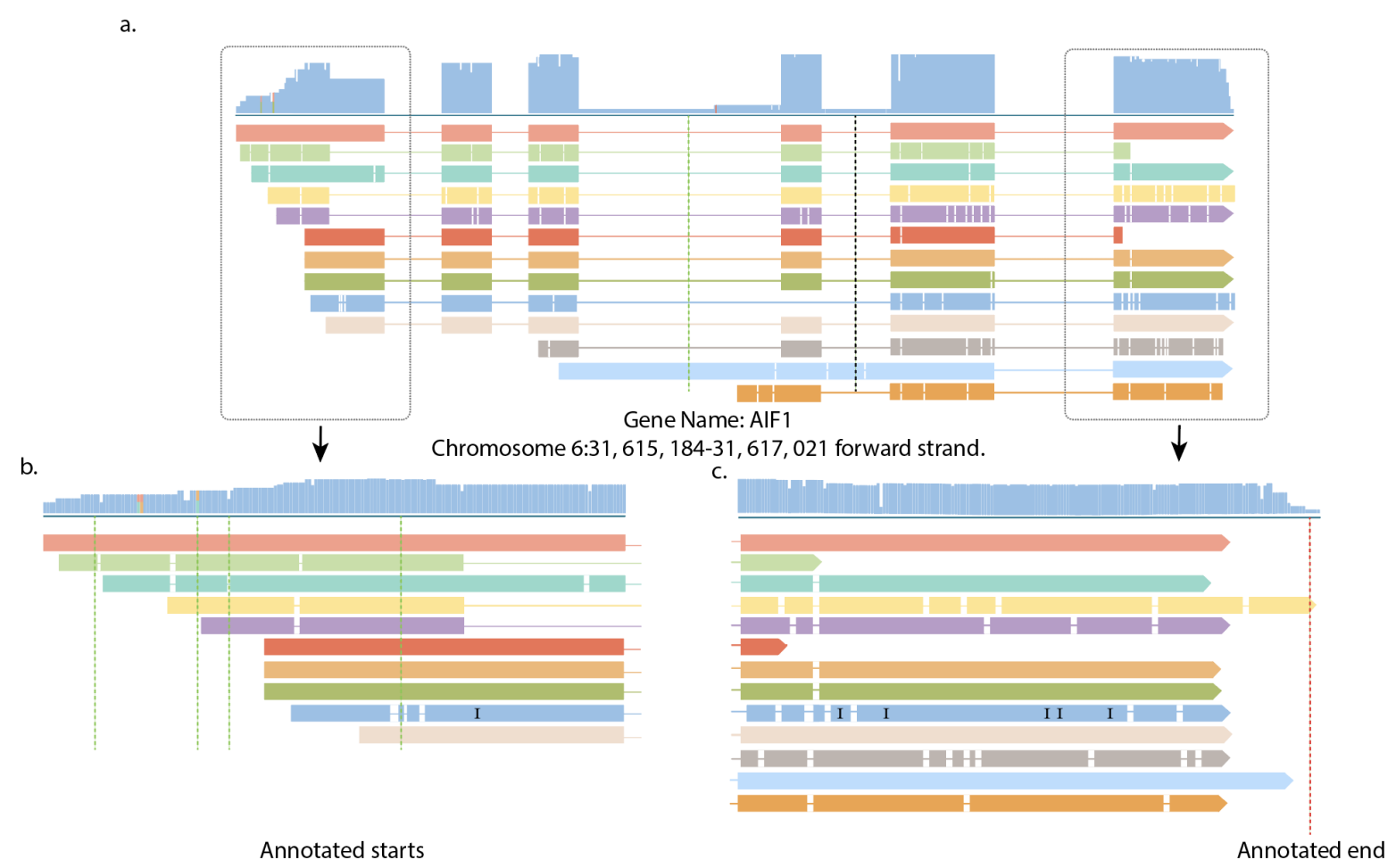

Figure 2: Variation of transcription start and end positions of consensus full-length transcripts.

(A) Thirteen multi-exon consensus full-length transcripts (CFL's) from gene AIF1 locus. (B) The first exon with vertical (green) lines demonstrating the location of annotated transcription start sites (TSS). Thirteen CFL's have a total of 13 different TSS, only 6 out of 13 TSS are within 10 bp of an annotated TSS. (C) The last exon with vertical (red) line shows the only annotated transcription end sites (TES). Thirteen CFL's have a total of 7 different TES, only 4 out of 7 TES are within 10 bp annotated TES. 


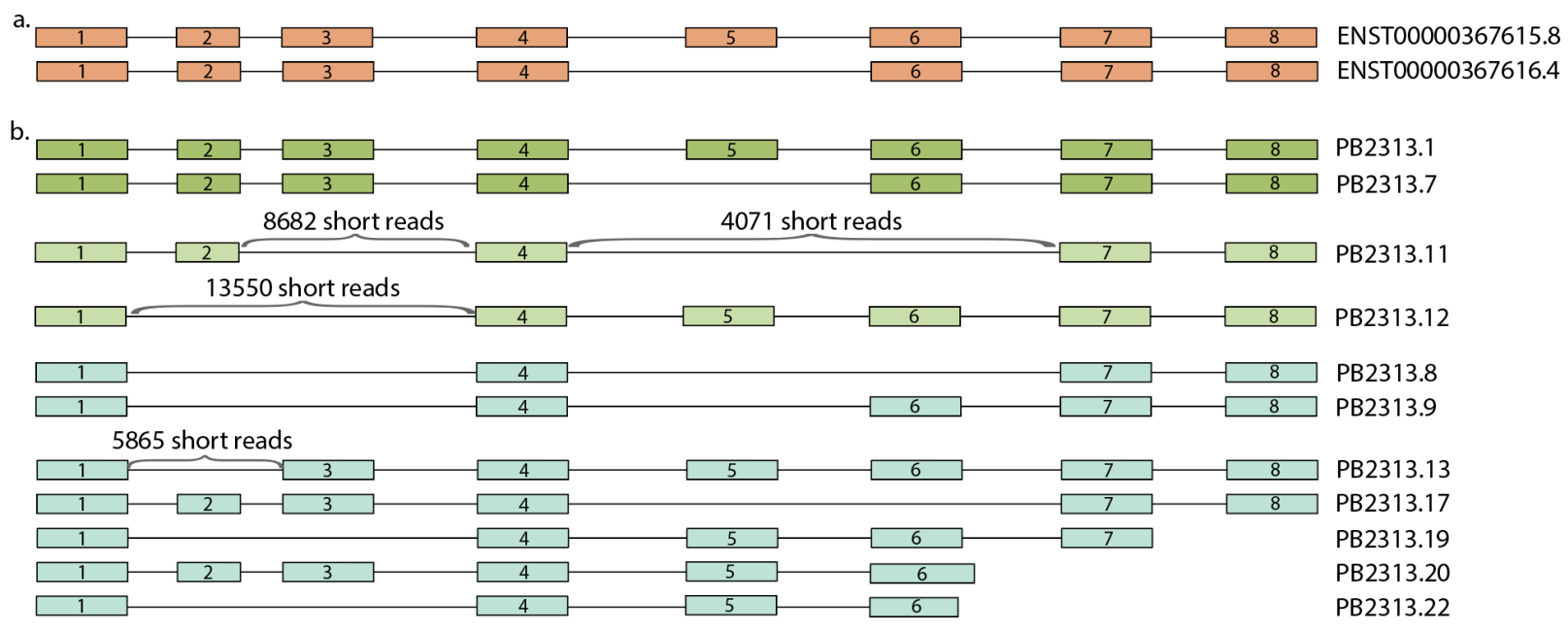

Figure 3: Gene model for podocin gene NPHS2. (a) Transcript structures of two annotated transcripts of NPHS2 in GENCODE. The second shorter annotated transcript is missing exon number 5. (b) Transcripts found in the PacBio glomerular sample and validated by our method. First two isoforms (dark green) match exactly to the annotated transcripts in GENCODE. Next two isoforms (light green) match two predicted transcript variants in NCBI's RefSeq annotation (XM_017002298.1 and XM_005245483.3). The remaining seven isoforms (gray) isoforms are potential novel transcript variants of NPHS2. The number of uniquely mapped reads to each of the novel junctions are noted above junctions. 


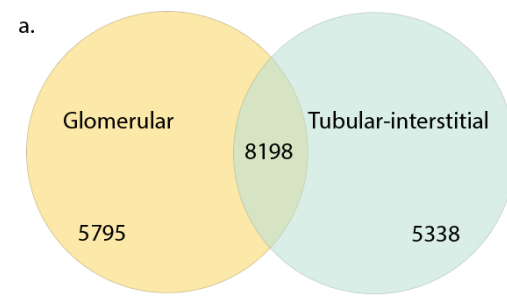

\begin{tabular}{|l|l|l|}
\hline \multicolumn{1}{|c|}{ KEGG pathway } & p-value \\
\hline \multirow{4}{*}{ Glomerular } & Rap1 signaling pathway & $2.9 \mathrm{e}-6$ \\
\cline { 2 - 3 } & Ribosome & $8.3 \mathrm{e}-6$ \\
\cline { 2 - 3 } & Non-alcoholic fatty liver disease (NAFLD) & $1.1 \mathrm{e}-4$ \\
\hline \multirow{4}{*}{ Tubular-interstitial } & Metabolic pathway & $4.8 \mathrm{e}-7$ \\
\cline { 2 - 3 } & Ubiquitin mediated proteolysis & $9.9 \mathrm{e}-5$ \\
\cline { 2 - 3 } & Aldosterone-regulated sodium reabsorption & $2.7 \mathrm{e}-4$ \\
\hline
\end{tabular}

c.

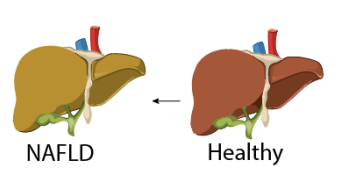

Non-alcoholic fatty liver disease (NAFLD)

Thyroid hormone signaling pathway
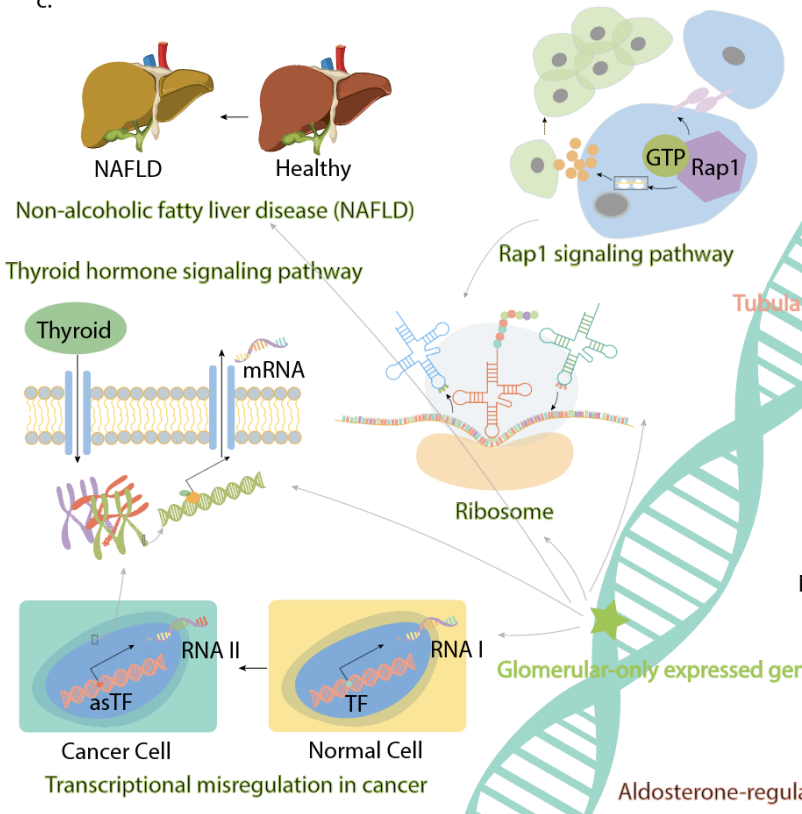

Rap1 signaling pathway
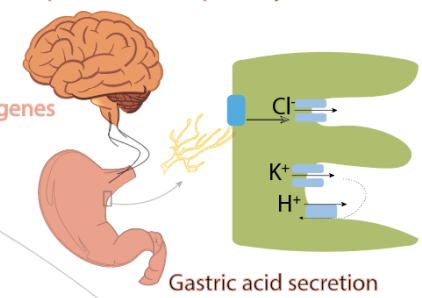

Ribosome
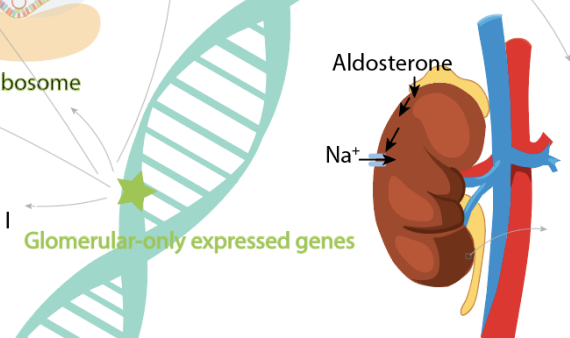

Ubiquitin Target protein

Proteolysis

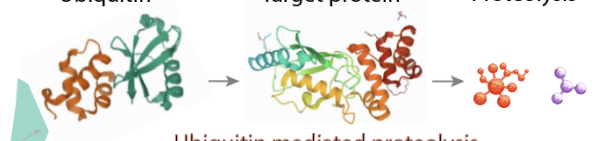

Ubiquitin mediated proteolysis

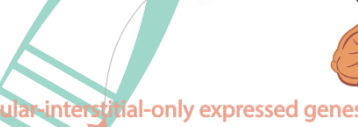


Table 1: Validation of PacBio transcript features from kidney tissue.

\begin{tabular}{|c|c|c|c|c|}
\hline & TSS & Splice Junctions & TES & Validated Multi-exon CFLs \\
\hline \multicolumn{5}{|l|}{ Glomerular } \\
\hline \multicolumn{5}{|l|}{ PacBio with Illumina ERCB } \\
\hline Annotation only & 3732 & 1194 & 4850 & 47785 \\
\hline Short reads only & & 5583 & 39 & \\
\hline Short reads or annotation & & 111506 & 5071 & \\
\hline Total & 38831 & 171742 & 26260 & 71492 \\
\hline \multicolumn{5}{|l|}{ Tubulointerstitial } \\
\hline \multicolumn{5}{|l|}{ PacBio with Illumina TN } \\
\hline Annotation only & 3534 & 7329 & 5278 & 53540 \\
\hline Short reads only & & 3105 & 8 & \\
\hline Short reads or annotation & & 119932 & 5318 & \\
\hline \multicolumn{5}{|l|}{ PacBio with Illumina ERCB } \\
\hline Annotation only & & 516 & 4883 & \\
\hline Short reads only & & 8824 & 104 & \\
\hline Short reads or annotation & & 125651 & 5425 & \\
\hline Total & 42896 & 185185 & 24625 & 75573 \\
\hline
\end{tabular}

TSS- transcription start site; TES- transcription end site; CFL consensus full-length transcripts; ERCB European renal cDNA bank; TN tumor nephrectomy. 
Table 2: Classification of validated-collapsed isoforms to GENCODE annotation using the Cuffcompare tool.

\begin{tabular}{|c|c|c|c|}
\hline & Type of Match & Validated Tubulo--interstitial Isoforms & Validated Glomerular Isoforms \\
\hline 1 & $\begin{array}{l}\text { Complete match of intron chain } \\
\text { with an annotated isoform }\end{array}$ & 10407 & 10882 \\
\hline 2 & $\begin{array}{c}\text { Contained within a reference } \\
\text { isoform }\end{array}$ & 3852 & 3857 \\
\hline \multicolumn{2}{|r|}{ Total annotated transcripts } & 14259 & 14739 \\
\hline 3 & Potentially novel isoform & 8910 & 7767 \\
\hline 4 & Intergenic transcript & 4208 & 9501 \\
\hline \multicolumn{2}{|r|}{ Total novel transcripts } & 13118 & 17268 \\
\hline 5 & $\begin{array}{l}\text { A transfrag falling entirely } \\
\text { within a reference intron }\end{array}$ & 11407 & 16627 \\
\hline 6 & $\begin{array}{c}\text { Single exon transfrag } \\
\text { overlapping a reference exon } \\
\text { and at least } 10 \text { bp of a reference } \\
\text { intron }\end{array}$ & 3729 & 4956 \\
\hline 7 & $\begin{array}{l}\text { Exonic overlap with reference } \\
\text { on the opposite strand }\end{array}$ & 2310 & 3420 \\
\hline
\end{tabular}




\section{References}

Alberts B, Johnson A, Lewis J, Raff M, Roberts K, Walter P. 2002. Studying Gene Expression and Function. Garland Science.

Dobin A, Davis CA, Schlesinger F, Drenkow J, Zaleski C, Jha S, Batut P, Chaisson M, Gingeras TR. 2013. STAR: ultrafast universal RNA-seq aligner. Bioinformatics 29: 15-21.

Eberwine J, Sul J-Y, Bartfai T, Kim J. 2014. The promise of single-cell sequencing. Nat Methods 11: $25-27$.

Frankish A, Diekhans M, Jungreis I, Lagarde J, Loveland JE, Mudge JM, Sisu C, Wright JC, Armstrong J, Barnes I, et al. 2021. GENCODE 2021. Nucleic Acids Res 49: D916-D923.

Gonzalez-Ibeas D, Martinez-Garcia PJ, Famula RA, Delfino-Mix A, Stevens KA, Loopstra CA, Langley CH, Neale DB, Wegrzyn JL. 2016. Assessing the Gene Content of the Megagenome: Sugar Pine (Pinus lambertiana). G3 6: 3787-3802.

Hosoya T, Li H, Ku C-J, Wu Q, Guan Y, Engel JD. 2018. High throughput single cell sequencing of both T-cell-receptor-beta alleles. http://dx.doi.org/10.1101/320614.

Howe KL, Achuthan P, Allen J, Allen J, Alvarez-Jarreta J, Amode MR, Armean IM, Azov AG, Bennett R, Bhai J, et al. 2021. Ensembl 2021. Nucleic Acids Res 49: D884-D891.

Ju W, Eichinger F, Bitzer M, Oh J, McWeeney S, Berthier CC, Shedden K, Cohen CD, Henger A, Krick $\mathrm{S}$, et al. 2009. Renal gene and protein expression signatures for prediction of kidney disease progression. Am J Pathol 174: 2073-2085.

Kato M, Wang M, Chen Z, Bhatt K, Oh HJ, Lanting L, Deshpande S, Jia Y, Lai JYC, O'Connor CL, et al. 2016. An endoplasmic reticulum stress-regulated lncRNA hosting a microRNA megacluster induces early features of diabetic nephropathy. Nat Commun 7: 12864.

Lea-Henry TN, Carland JE, Stocker SL, Sevastos J, Roberts DM. 2018. Clinical Pharmacokinetics in Kidney Disease: Fundamental Principles. Clin J Am Soc Nephrol 13: 1085-1095.

Li H-D, Menon R, Omenn GS, Guan Y. 2014. The emerging era of genomic data integration for analyzing splice isoform function. Trends Genet 30: 340-347.

McManus CJ, Graveley BR. 2011. RNA structure and the mechanisms of alternative splicing. Curr Opin Genet Dev 21: 373-379.

O’Grady T, Wang X, Höner Zu Bentrup K, Baddoo M, Concha M, Flemington EK. 2016. Global transcript structure resolution of high gene density genomes through multi-platform data integration. Nucleic Acids Res 44: e145.

O’Leary NA, Wright MW, Brister JR, Ciufo S, Haddad D, McVeigh R, Rajput B, Robbertse B, Smith-White B, Ako-Adjei D, et al. 2016. Reference sequence (RefSeq) database at NCBI: current status, taxonomic expansion, and functional annotation. Nucleic Acids Res 44: D733-45.

Roberts A, Pimentel H, Trapnell C, Pachter L. 2011a. Identification of novel transcripts in annotated 
genomes using RNA-Seq. Bioinformatics 27: 2325-2329.

Roberts A, Trapnell C, Donaghey J, Rinn JL, Pachter L. 2011b. Improving RNA-Seq expression estimates by correcting for fragment bias. Genome Biol 12: R22.

Saran R, Robinson B, Abbott KC, Bragg-Gresham J, Chen X, Gipson D, Gu H, Hirth RA, Hutton D, Jin Y, et al. 2020. US Renal Data System 2019 Annual Data Report: Epidemiology of Kidney Disease in the United States. Am J Kidney Dis 75: A6-A7.

Schmid H, Boucherot A, Yasuda Y, Henger A, Brunner B, Eichinger F, Nitsche A, Kiss E, Bleich M, Gröne H-J, et al. 2006. Modular activation of nuclear factor-kappaB transcriptional programs in human diabetic nephropathy. Diabetes 55: 2993-3003.

Sharon D, Tilgner H, Grubert F, Snyder M. 2013. A single-molecule long-read survey of the human transcriptome. Nat Biotechnol 31: 1009-1014.

Steijger T, Abril JF, Engström PG, Kokocinski F, RGASP Consortium, Hubbard TJ, Guigó R, Harrow J, Bertone P. 2013. Assessment of transcript reconstruction methods for RNA-seq. Nat Methods 10: $1177-1184$.

Trapnell C, Hendrickson DG, Sauvageau M, Goff L, Rinn JL, Pachter L. 2013. Differential analysis of gene regulation at transcript resolution with RNA-seq. Nat Biotechnol 31: 46-53.

Trapnell C, Williams BA, Pertea G, Mortazavi A, Kwan G, van Baren MJ, Salzberg SL, Wold BJ, Pachter L. 2010. Transcript assembly and quantification by RNA-Seq reveals unannotated transcripts and isoform switching during cell differentiation. Nat Biotechnol 28: 511-515.

Wang Z, Gerstein M, Snyder M. 2009. RNA-Seq: a revolutionary tool for transcriptomics. Nat Rev Genet 10: $57-63$.

Weirather JL, Afshar PT, Clark TA, Tseng E, Powers LS, Underwood JG, Zabner J, Korlach J, Wong WH, Au KF. 2015. Characterization of fusion genes and the significantly expressed fusion isoforms in breast cancer by hybrid sequencing. Nucleic Acids Res 43: e116.

Wu TD, Watanabe CK. 2005. GMAP: a genomic mapping and alignment program for mRNA and EST sequences. Bioinformatics 21: 1859-1875. 